\title{
Envelope protein as molecular target for YFV 17D (yellow fever virus) Drug discovery
}

M. Muni Kumar*, D. Pradhan, I.V. Priyadarshini, A. Umamaheswari** and G. Subramanyam SVIMS Bioinformatics Centre, Department of Bioinformatics, SVIMS University, Tirupati -517507, India. Email: svims.btisnet@nic.in

\section{Key Message}

Yellow fever (YF), a mosquito-borne (Aedes aegypti) viral haemorrhagic fever, is one of the most lethal viral diseases. Despite the availability of vaccines, yellow fever virus (YFV) strikes an estimated 2, 00,000 persons world-wide each year and causes 30,000 deaths approximately (Schmaljohn, et al., 1996). There are no approved antiviral therapies for the treatment of YF in human (Tomori, et al., 2004). YFV 17 strain R cleotides, which encodes three structural proteins (C, PrM, and E) and seven nonstructura proteins (NS1, NS2A, NS2B, NS3, NS4A, NS4B, and NS5).

Identification of different protein functions facilitates a mechanistic understanding of YFV infection and opens novel means for drug development. Functional assignment of complet YFV 17D proteome was done through support Vector machine (SVM) (Sahoo et al. 2008), The envelope (E) protein is the major structural protein of YFV17D and is understood to mediate several important virus functions such as host cell receptor-binding, membrane fusio activity and virion assembly (Heinz \& Mandl, 1993). EMBOSS antigenic server (Dass et al 2008) and Pfam analysis (Eddy et al., 1997) deciphered E protein is the major antigen responsible for induction of neutralizing antibodies and protective immunity in host. The YFV 17D E protein being non-homologous to human, we propose it as molecular target for structure based drug discovery (SBDD) against YFV 17D.

3D structure of YFV 17D E protein was built based on crystal structure of Dengue virus $2 \mathrm{M}$ jor envelope protein in MODELLER9V7 (Sahoo et al., 2009). Six selected lead molecules were docked to functionally important binding site of YFV 17D E protein using LigandFi (Sahoo et al, 2009) module of Discovery Studio 2.0. Heparin docked into the receptor bind441 and further ADME (Absorption, Disruption, Metabolism and Excretion) studies.

Material and Methods

Whole proteome was retrieved from NCBI Ref seq FTP server

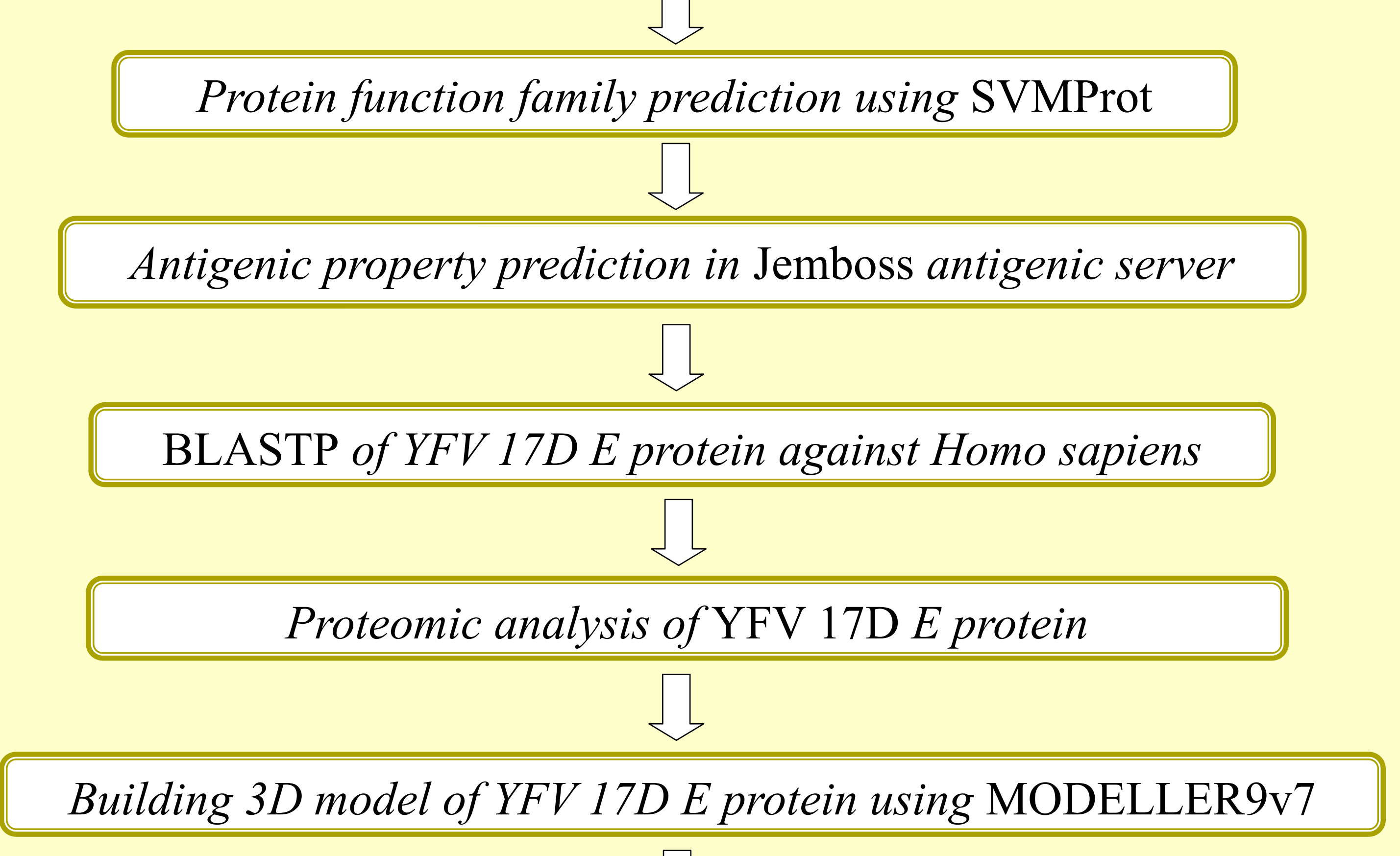

Building 3D model of YFV 17D E protein using MODELLER9v7

\section{Model validation using PROCHECK}

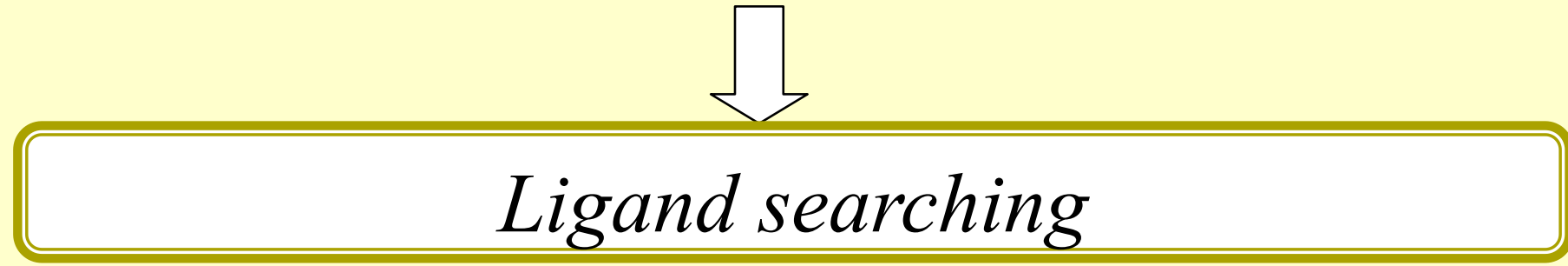

Ligand searching

Protein - Ligand Docking using the LigandFit of Dis-
Results and Discussion

YFV proteome consists of 13 proteins. Structural and non-structural protein functions are metal -binding, lipi binding, copper-binding, calcium-binding, zinc-binding, magnesium-binding, DNA-binding, RNA-binding, transmembrane and outer membrane group of proteins.

The YFV 17D E protein comprising of 493 amino acids and is among the family of metal-binding, outer membrane, the transmembrane protein, aptamer-binding protein, P-type ATPase - (P-ATPase) family, zinc-binding and coat protein. The functional families reveals its importance in receptor binding, hemagglutination of erythrocytes at acidic phe induction of the protective immune response, and involvement in an intraendosomal acid-cotalyzed fusion step necessary for infection.

Twelve antigenic sites were predicted in YFV 17D E protein using EMBOSS antigenic server. Presence of more antigenic sites is expected to initiate strong immunogenic response in host.

. The E protein being a human non-homolog, plays an active role in viral infection and due to the presence of more antigenic sites $\mathrm{E}$ protein was selected as a potent molecular target against $\mathrm{YFV}$ drug discovery.

Pfam analysis revealed YFV 17D E protein having domains for induction of neutralizing antibodies and protecti

Three Dimensional structure of YFV 17 D E protein was built based on crystal structure of Dengue virus 2 Major envelope protein (PDB ID: 1P58) in MODELLER9V7. The model evaluation using PROCHECK revealed 97.7\% residues are in favorable region (Fig.1). The model was visualized through RASMOL (Fig.2). The structure was energy minimized (-7.95 kcal/mol) using CHARMm.

Twenty nine selected ligand molecules specific for envelope protein were downloaded and prepared using LigandFi (Lee et al 2004). Only six ligand molecules seemed to follow Lipinski's rule of five.

The six prepared ligand molecules were docked to functionally important binding site of YFV 17D E protein using LigandFit (Sahoo et al., 2009) module of Discovery Studio 2.0. Heparin docked into the receptor binding site with dock score of 42.11 shown in table 1, and four hydrogen bonds (one each with SER 483, ALA 441 and two with GLN 443) in sixth pose shown in Fig. 3A and 3B. The residues such as ALA441, PHE442, LUE487 and VAL491 are forming hydrophobic pocket while GLN250, GLU251, ASN62 and ASN259 are forming hydrophilic surface around the active site. These residues were involved in hydrophobic and hydrophilic interaction respectively with the ligand molecule

The residues involved in hydrogen bond are GLY 433, ALA 441 and SER 488. Hydrophobic residues (ALA441, PHE442 and LEU 487) and hydrophilic residues (ASN62) of YFV 17D E protein involved in docking interactio with heparin are also part of an epitope identified using Jemboss. So, based on the results obtained through the docking studies we can conclude that heparin can be used as a lead molecule. Further ADME (Absorption, Disruption, Metabolism and Excretion) studies to confirm the activity of the molecule which needs to be tested for biological activity before entering into human clinical trials.

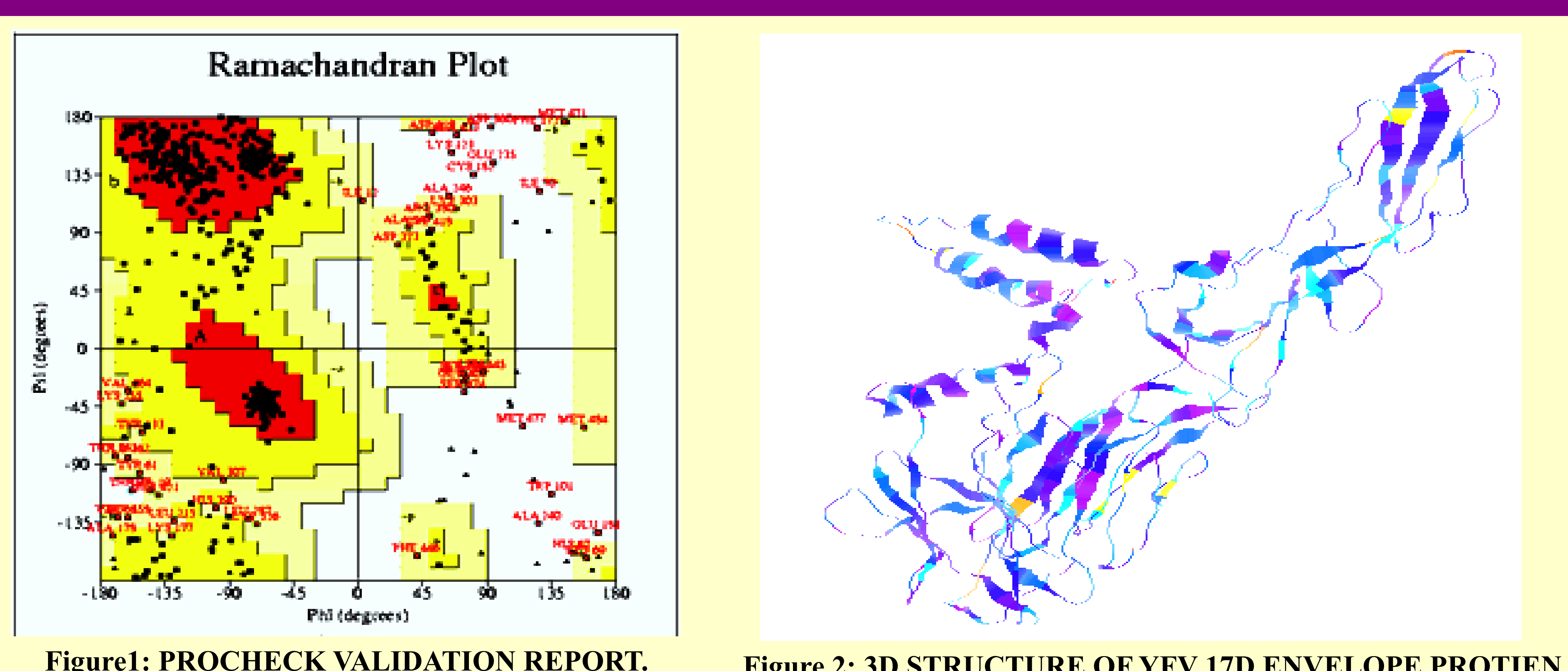

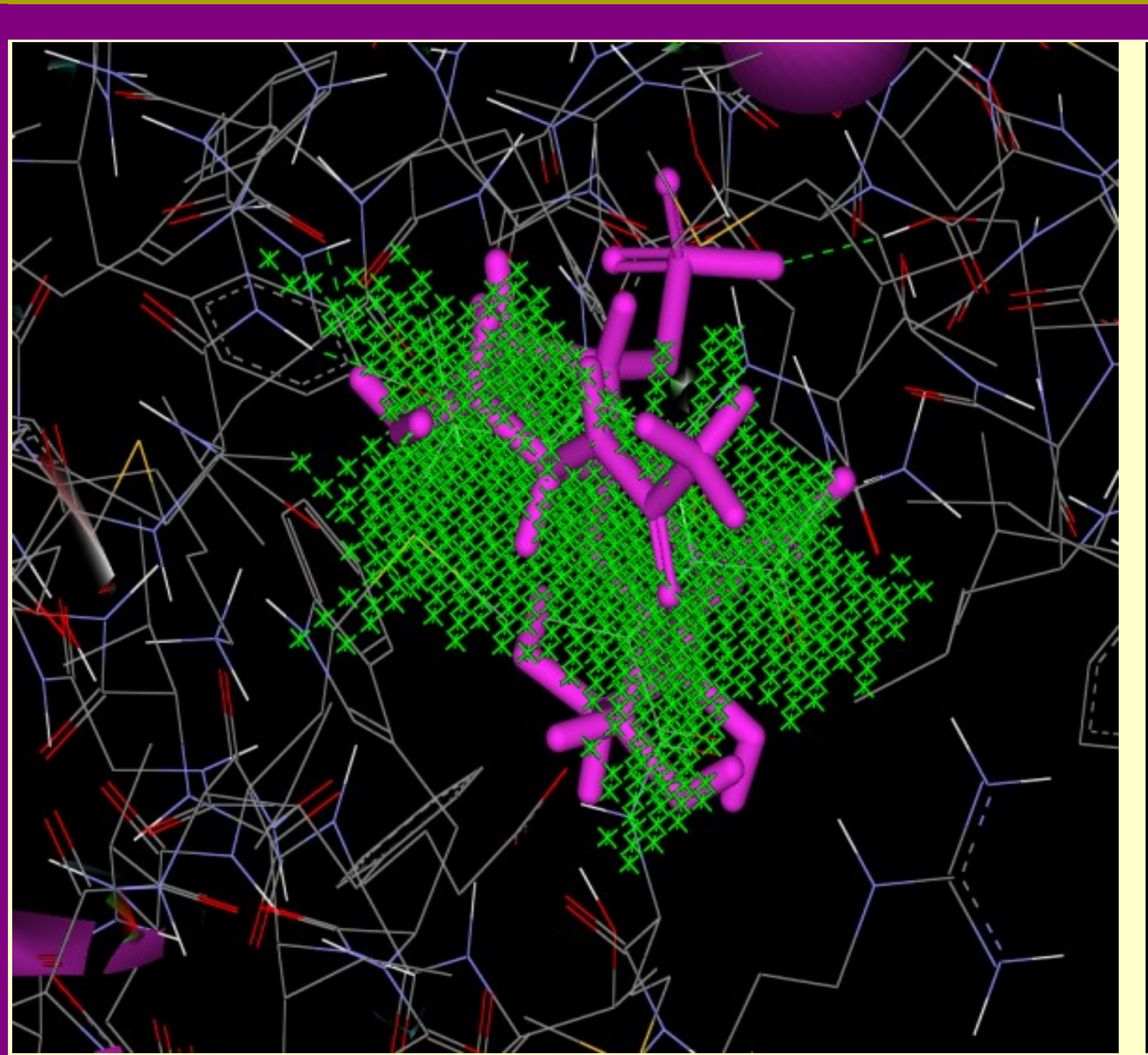

Figure 3A: HEPARIN DOCKED WITH
ENVELOPE PROTEIN OF YFV 17D

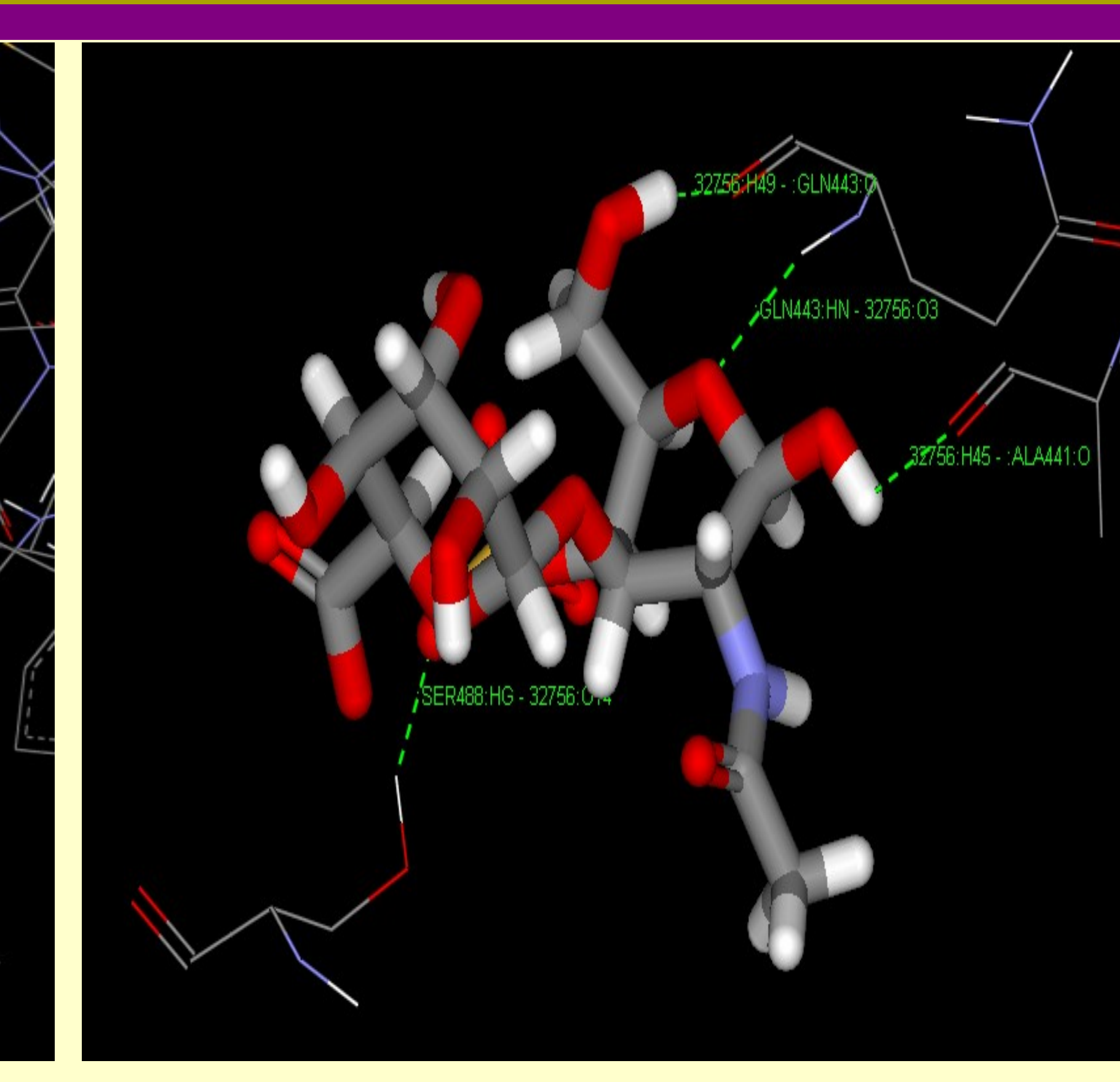

FIGURE 3B: HYDROGEN BONDS BETWEEN
THE TARGET PROTEIN AND THE LIGAND

\begin{tabular}{|l|c|}
\hline \multicolumn{2}{|c|}{ DOCKING RESULTS } \\
\hline Number of binding sites & 14 \\
\hline Number of poses found & 10 \\
\hline Clustering the final poses & 6 \\
\hline Best docking score & 42.11 \\
\hline Best Ligand Pose : energy & $-7.95 \mathrm{kcal} / \mathrm{mol}$ \\
\hline Number of Hydrogen bonds & 4 \\
\hline
\end{tabular}

CONCLUSION

The protein function family was identified common to YFV 17D proteome would facilita mechanistic understanding of YFV infection and opens novel means for drug development. The YFV 17D E protein was important role in receptor binding, hemagglutination of erythrocytes at acidic $\mathrm{pH}$, induction of the protective immune response, and involvement in an intraendosomal acid-catalyzed fusion step necessary for infection.

The predicted 3D model of YFV 17 D E protein is of good quality. Hence the functional domain of the protein could be used for structure based virtual screening in order to obtain more ligands.

The lead candidate heparin blocks the functionally important site of YFV 17D E protein by forming hydrogen bonds, hydrophobic, hydrophilic and electrostatic interaction. Hence, the heparin would be considered as a good drug molecule.

cknowledgements: We are grateful to DBT, Ministry of Science and Technology, Govt. of India, for financial support. 